\title{
Treizième réunion de l'AISRU. Székesfehervar (Hongrie) 12-14 mai
} 1982

Denise Pumain

Citer ce document / Cite this document :

Pumain Denise. Treizième réunion de l'AISRU. Székesfehervar (Hongrie) 12-14 mai 1982. In: Population, $37^{e}$ année, $n^{\circ} 6$, 1982. pp. 1211-1214;

http://www.persee.fr/doc/pop_0032-4663_1982_num_37_6_1311

Document généré le 10/06/2016 


\title{
COMPTE RENDUS DE COLLOQUE
}

\section{TREIZIÈME RÉUNION DE L'AISRU}

\author{
Székesfehervar (Hongrie) 12-14 mai 1982
}

La treizième réunion de l'Association Internationale pour la Statistique Régionale et Urbaine s'est déroulée autour de trois thèmes. Le premier, organisé par M. Barabas (Institut National Hongrois de Statistiques), concernait «l'étude statistique des systèmes de peuplement». Les communications ont permis de caractériser les principaux problèmes contemporains posés par l'organisation du réseau urbain en Hongrie. Les intervenants ont souligné les similitudes entre le processus d'urbanisation hongrois et celui des pays industrialisés. Les particularités actuelles du réseau (taux d'urbanisation «moyen »: $53 \%$ en 1980, forte concentration de la population dans la capitale : Budapest représente $19 \%$ de la population totale en 1980 , plus de 8 fois la population de la $2^{\prime \prime}$ ville) s'expiiquent par le caractère tardif de l'industrialisation du pays, qui n'a induit une croissance urbaine rapide qu'après 1945, par la trame ancienne du peuplement rural, dense, faite de fermes dispersées et de très gros villages, et par une forme de développement d'abord très centralisée. La croissance de la population urbaine n'est pas très forte (un peu plus de $1 \%$ par an entre 1970 et 1980 , contre environ $0,3 \%$ pour la population totale). Elle s'est beaucoup ralentie à Budapest par suite d'une politique de décentralisation industrielle et profite davantage aux 23 villes chefs-lieux de divisions administratives, à de petites villes de banlieue ou de régions touristiques, ainsi qu'aux villages récemment promus au statut urbain. La part de la migration dans cet accroissement tend à diminuer, elle représente encore entre la moitié et les deux-tiers et tend à rajeunir la population des villes dont la croissance est la plus forte. Mais le fait à souligner est l'importance considérable des navettes: sur 5 millions d'actifs, 2 millions sont des navetteurs (45\% de la population active des villages se déplace chaque jour pour son travail). Ce va-et-vient permanent va de pair avec le très bas prix des transports, la crise du logement urbain, et la fréquence d'un complément d'activité agricole $(2 / 5$ des ménages cultivent un lopin familial, et cette activité procure plus de $1 / 5$ du revenu total des villages). Une base de données sur l'ensemble des communes hongroises est désormais mise à jour annuellement, et comporte des informations plus détaillées (plus de 200 variables) sur les 150 unités les plus peuplées. Elle est utilisée pour la prévision des besoins liés à l'urbanisation et aussi pour l'attribution du statut de ville. C'est, en effet, une combinaison complexe de critères objectifs et normatifs (économiques, culturels, administratifs, urbanistiques...) qui définit, en Hongrie, le caractère urbain d'une unité de peuplement, conféré par une décision gouvernementale. Le nombre des villes est ainsi passé de 54 en 1949 à 96 en 1980. En dehors 
de la dizaine d'agglomérations ou de régions urbaines qui dépassent 100000 habitants, la plupart d'entre elles n'ont que de 15 à 30000 habitants, dimension déjà atteinte par près de cinquante autres unités de peuplement demeurées rurales.

Le deuxième thème, "transport urbain, structures résidentielles et localisation des emplois », était organisé par L.H. Klaassen (Netherlands Economic Institute). La plupart des études de transport prennent comme point de départ une structure spatiale des villes définie par la localisation de l'habitat, des lieux de travail et de prestations de services. Le dessin du réseau et les prévisions de trafic sont déduits de cette structure. Cette clémarche a été illustrée par la présentation détaillée d'une enquête concernant les déplacements quotidiens de la population à Budapest. D'autres approches ont été proposées, pour tenir compte des effets de l'évolution des modalités de transport sur la configuration des résidences et des activités. Plusieurs communications plus théoriques se sont attachées 1) à mettre en relation divers types de structure spatiale et leur développement avec les modèles de mouvement (dans le cadre des cycles d'évolution urbaine définis par L. Klaassen : urbanisation, suburbanisation, désurbanisation, réurbanisation); 2) à souligner l'intérêt d'une planification intégrée reliant systématiquement les modifications du système de transports et celles de la structure spatiale des villes; 3) à évaluer l'influence à long terme d'un accroissement des coûts de transport sur la structure spatiale des régions urbaines (qui combinerait une tendance locale à la concentration dans les zones déjà agglomérées, et une tendance globale à la déconcentration, par un accroissement des villes petites et moyennes plus rapide que celui des très grandes); 4) à proposer une méthode permettant d'identifier et de délimiter, au sein d'un espace urbain fortement centralisé, des zones secondaires d'échange privilégié, noyaux possibles d'une éventuelle restructuration des déplacements à l'intérieur d'une agglomération.

Le troisième thème: "Observation et description des migrations et des déplacements temporaires à l'aide d'enquêtes » était organisé par D. Courgeau. L'objectif était de mettre au point des techniques comparables pour saisir et étudier des formes de mobilité que les statistiques usuelles ne prennent pas bien en compte. Malgré la diversité des exemples présentés, la discussion fut très générale et l'accord se fit sur plusieurs points. En ce qui concerne les techniques d'enquête dans les pays développés, il semble que les pays ayant des registres de population (Danemark, Belgique...) disposent de la meilleure information relative aux migrations. Cependant, cet avantage concerne seulement la mesure du phénomène et non l'étude de ses composantes ou des motivations des migrants, qui requiert des enquêtes complémentaires. En URSS, le double enregistrement des migrations, au départ et à l'arrivée, permet de bonnes évaluations globales (certains mouvements intrarégionaux n'étaient, toutefois, pas pris en compte jusqu'ici par ce système). En l'absence de registre de population, la méthode préconisée par $\mathrm{Ph}$. Collomb pour reconstituer l'ensemble des migrations intervenues à partir d'une zone rurale apparut comme très efficace. En ce qui concerne l'analyse des motifs de la migration, l'accent fut mis sur l'intérêt qu'il y aurait à réaliser des enquêtes non plus seulement rétrospectives, mais aussi prospectives, pour faire la part entre les motivations 
réelles, les avantages attendus et les rationalisations a posteriori. Enfin, dans la mesure où les migrations s'intensifient à l'intérieur des zones urbanisées et tendent à diminuer entre les grandes régions (exemples cités en Belgique, RDA, Suisse, Tchécoslovaquie, Bulgarie), tandis que se modifient les limites des unités territoriales où elles sont comptabilisées, il semble souhaitable d'adopter, comme définition la plus générale possible de la migration, le changement de logement. Certains pays s'emploient à distinguer dans les recensements le caractère principal ou secondaire, permanent ou temporaire du ou des logements déclarés par un individu. Cette nécessité d'asseoir les enquêtes sur des bases conceptuelles claires apparut encore avec plus d'acuité dans la présentation des exemples pris en pays sous-développés : alors que les techniques pour la mesure des migrations (passages successifs, remontée des filières de migration) sont à peu près partout transposables, les notions permettant de définir les migrations temporaires ou définitives sont beaucoup plus difficiles à comparer. Ces difficultés imposent de reconsidérer le phénomène migratoire dans la perception que chaque culture a de l'espace, qui délimite les divers types de mobilité possibles, en un lieu et à un moment donnés. Cette perspective générale doit guider la conception des enquêtes que lon est trop souvent tenté de limiter aux modalités de déplacement le plus souvent observées dans le pays et pour la période que l'on étudie.

Au total, le contenu de la réflexion et les échanges intervenus lors de ces journées particulì̀rement denses, vivifiées par la qualité de l'accueil hongrois, laissent bien espuer des futures réunions de l'Association.

\title{
Denise Pumain.
}

\author{
LISTE DES COMMUNICATIONS \\ (d'après la table des matières des 3 volumes \\ de comptes rendus publiés par l'AISRU)
}

B. Barta (Hongrie), - Aspects sociaux et écologiques du processus d'urbanisation.

G. BORA (Hongrie). - Place et fonction des différents types d'habitat dans le réseau de peuplement.

G. Vukovich (Hongrie). - Importance de l'approche sociologique dans la formation de l'espace habité.

G. Koszegfalvi (Hongrie). - Les changements structuraux du système de peuplement hongrois.

T. Kovacs (Hongrie). - Méthodes statistiques d'analyse du réseau de peuplement.

L. Van Den Berg, L.H. Klaassen, J. Van Der Meer (Pays-Bas). - Structures spatiales et transports dans les régions urbaines.

J. Bourdrez (Pays-Bas). - Politique urbaine et système de transport dans une structure physique changeante.

D. Pumain (France). Composition des flux migratoires et structures socioéconomiques des villes.

R. Funck, U. Blum (RFA). - Influence de l'accroissement récent des coûts de transport sur les mouvements dans les zones urbaines (court terme) et sur leur structure spatiale (long terme). 
R. Nagy, M. Lelkes (Hongrie). - L'enquête de trafic 1974-1975 et les projections de trafic pour Budapest.

J.J. RONSAC (France). — Zones de solidarité en région d'lle-de-France.

Ph. Collomb (France). - Les émigrants de l'Ouest audois, 19 ans après. Une enquête sur la migration interne rurale dans un pays céréalier du sud de la France, méthodes pratiques de collecte d'une information utile pour les monographies de migration régionale.

C.A. Wol.. (Danemark). - La réalisation de l'enquête de 1979 et, particulièrement, de l'élaboration du questionnaire.

G.W. SMITH (Royaume-Uni). - Enquêtes de migration au Royaume-Uni.

J. Vaugrlade, S.P. Coulibaly (France). - Observation et description des migrations et des déplacements temporaires: le cas de la Haute-Volta.

C.H. Triller (Etats-Unis). - Migration temporaire et saisonnière en Amérique Centrale: approche conceptuelle et méthodologique de sa mesure.

B. HANNA (Thailande). - L'approche de l'ESCAP pour les enquêtes de migration.

J. Dvorzak, J. Gavelka (Tchécoslovaquie). - Problèmes des enquêtes de statistiques démographiques et changements de résidence temporaire en Tchécoslovaquie.

R.M. Dimitrieva (URSS). - Etude statistique de la migration en URSS.

M. Fleury (France). - Présentation générale de l'enquête sur les mobiles de
migration.

M. Picouet (France). - Deux méthodes d'enquêtes originales utilisées en Afrique pour l'observation et la description des migrations.

S. TAsev, P. Bozikov (Bulgarie). - L'examen de la migration interne et des navettes à l'occasion du recensement de la population de la Bulgarie en 1975.

\section{LA CHAIRE QUETELET}

\section{Louvain-la-Neuve, 4 au 7 mai 1982}

La Chaire Quetelet, organisée chaque année par le Département de Démographie de l'Université Catholique de Louvain, s'est tenue à Louvain-laNeuve du 4 au 7 mai 1982 sur le thème de «La morbidité et la mortalité aux âges adultes dans les pays développés».

Articulées autour d'une ou deux communications sollicitées, les séances ont été en grande partie consacrées à la discussion, permettant aux différents participants, pour la plupart européens, d'échanger leurs expériences.

Yves Peron " 1 ' faisant le point sur l'évolution récente de la morbidité et de la mortalité des adultes a brossé la toile de fond du colloque : après l'aggravation de la mortalité masculine entre 35 et 65 ans qui a marqué les années soixante, on assiste actuellement à une stabilisation voire à un nouveau recul de la mortalité dans la plupart des pays européens (à l'exception des pays de l'Europe de l'Est) et surtout dans les pays anglo-saxons d'outre-mer

(1) Tendances récentes de la morbidité et de la mortalité à l'âge adulte dans les pays développés. 